Supporting Information

\title{
Catalytic Reductive Alkylation of Amines in Batch and Microflow Con- ditions Using a Silicon-Wafer-Based Palladium Nanocatalyst
}

Takuma Sato, ${ }^{1}$ Yasuhiro Uozumi, ${ }^{1,2}$ and Yoichi M. A. Yamada ${ }^{*}$

${ }^{1}$ RIKEN Center for Sustainable Resource Science, Wako, Saitama 351-0198, Japan

2Institute for Molecular Science (IMS), Myodaiji, Okazaki, Aichi 444-8787, Japan 
General information. All synthetic manipulations were carried out under ambient conditions unless otherwise stated. Reagents and solvents were obtained from commercial suppliers and used as received unless otherwise stated. $\mathrm{Pd} / \mathrm{C}(5 \mathrm{wt} \%)$ and $\mathrm{Pd} / \mathrm{Al}_{2} \mathrm{O}_{3}(5 \mathrm{wt} \%)$ were obtained from Wako Pure Chemical Industries and Aldrich, respectively. Polyethylene glycol monostearate ( $\mathrm{n} \approx 55$, containing palmitate) was purchased from Tokyo Chemical Industry (TCI). Hydrofluoric acid (46\%, JIS Special Grade) and hydrogen peroxide (30\%, Super Special Grade), $\mathrm{HAuCl}_{4} \cdot 3 \mathrm{H}_{2} \mathrm{O}$, and $\left(\mathrm{NH}_{4}\right)_{2} \mathrm{PdCl}_{4}$ were purchased from Wako Pure Chemical Industries. The p-type Si(100) wafer (B-doped, 10-100 $\Omega \bullet \mathrm{cm}$, double-side-polished, 2-inch, $300 \mu \mathrm{m}$ thick) was purchased from Electronics and Materials Corporation. Water was purified by Millipore Direct-Q UV system. Dehydrated 2-propanol $\left(\mathrm{H}_{2} \mathrm{O}<10 \mathrm{ppm}\right)$ was purchased from Wako Pure Chemical Industries. Molecular sieve (pore size $0.3 \mathrm{~nm}$, pellet) was purchased from Wako Pure Chemical Industries and dried at $300{ }^{\circ} \mathrm{C}$ for $2 \mathrm{~h}$ under reduced pressure prior to use. $\mathrm{H}_{2}$ (purity $>99.99999 \%, \mathrm{O}_{2}<$ $0.02 \mathrm{ppm}$, dew point $<-80{ }^{\circ} \mathrm{C}$ ) and $\mathrm{Ar}$ (purity $>99.999 \%, \mathrm{O}_{2}<0.2 \mathrm{ppm}$, dew point $<-70{ }^{\circ} \mathrm{C}$ ) gas cylinders were obtained from Taiyo Nippon Sanso Corporation and used for flow reactions. ${ }^{1} \mathrm{H}$ NMR (400 MHz) and ${ }^{13} \mathrm{C}$ NMR (100 MHz) spectra were recorded with JEOL JNM-AL400. The ${ }^{1} \mathrm{H}$ NMR chemical shifts were reported relative to tetramethylsilane $(0.00 \mathrm{ppm})$ or residual $\mathrm{HDO}(4.65 \mathrm{ppm})$ in $\mathrm{D}_{2} \mathrm{O}$. The ${ }^{13} \mathrm{C}$ NMR chemical shifts were reported relative to $\mathrm{CDCl}_{3}(77.0 \mathrm{ppm}),\left(\mathrm{D}_{3} \mathrm{C}\right)_{2} \mathrm{SO}(39.5 \mathrm{ppm})$, or internal $\mathrm{CH}_{3} \mathrm{CN}$ (1.47 ppm) in $\mathrm{D}_{2} \mathrm{O}$. The GC analysis was performed by Agilent 6850 equipped with a capillary column (Supelco SLB-IL60, $30 \mathrm{~m} \times$ i.d. $0.25 \mathrm{~mm}$, df $0.20 \mu \mathrm{m}$ ). Melting point was measured by Round Science RFS-10. TLC analysis was performed on glass plates coated with silica gel (Merck TLC silica gel 60 $\left.\mathrm{F}_{254}\right)$ using UV light $(\lambda=254 \mathrm{~nm}), p$-anisaldehyde $/ \mathrm{H}_{2} \mathrm{SO}_{4}, \mathrm{I}_{2} / \mathrm{SiO}_{2}$, and a modified Dragendorff reagent (prepared using L-tartaric acid) for visualization. Column chromatography was carried out on silica gel (Wakogel C-200 (Wako Pure Chemical Industries)). 3-Aminopropyl-functionalized silica gel (Wakogel $50 \mathrm{NH}_{2}$ ) was purchased from Wako Pure Chemical Industries. Medium pressure liquid chromatography (MPLC) was performed using Yamazen W-Prep 2XY with a UV detector. Scanning transmission electron microscope (STEM) imaging was performed using JEOL JEM-2100F equipped an energy dispersive X-ray spectroscopy (EDS) detector. FIB milling was performed by Hitachi NB5000. FT-IR spectra were recorded by JASCO FT/IR-6200. HRMS spectra were recorded by Bruker MicrOTOF QIII in ESI or APCI mode using sodium formate clusters as a calibrant. Specific rotation was measured by JASCO P2200 equipped with a Peltier thermostated cell holder with Na D-line $(589 \mathrm{~nm})$ at $20.0{ }^{\circ} \mathrm{C}$. Chiral HPLC analysis was performed using JASCO LC-2000plus system equipped with a UV detector. UV-VIS absorption spectra were measured by JASCO V-650 at room temperature. ICP-MS analysis for ${ }^{106} \mathrm{Pd}$ was performed using PerkinElmer NexION 350D, where all samples were treated in high-purity $\mathrm{HNO}_{3}$ under microwave irradiation using PerkinElmer Titan MPS before analysis. The micro flow devices (catalyst housing) and the mass flow controller were manufactured by DFC Co., Ltd. (Uji, Kyoto, Japan). In the 
flow reaction system, the HPLC pump (FLOM Corporation; KP-22-01S) and the hotplate (MSA Factory Co., Ltd. (Tokyo, Japan); PA3020-H, PCC100F-10A, PCC-055-1M) were commercial products and used as received. 
Catalyst reusability experiment. To a glass tube was added 2-phenylethylamine (1b, $1.0 \mathrm{mmol})$, benzaldehyde (2a, $1.2 \mathrm{mmol})$, 2-propanol (2 mL), and SiNS-Pd (0.02 mol\%, ca. $160 \mathrm{mg})$. The reaction vessel was sealed with a rubber septum and purged with $\mathrm{H}_{2}$. The mixture was reacted under $\mathrm{H}_{2}(0.1 \mathrm{MPa})$ in a balloon at $60{ }^{\circ} \mathrm{C}$ for $18 \mathrm{~h}$ with vortex mixing at $400 \mathrm{rpm}$. After cooling to room temperature, the wafer was washed with EtOAc $(3 \times$ ca. $5 \mathrm{~mL})$ and the combined solution was concentrated under reduced pressure. The wafer was rinsed with $\mathrm{EtOH}$ (ca. $5 \mathrm{~mL}$ ), dried under air, and reused for the next reaction.

Leaching test. The concentrated crude material (206.7 mg) was dissolved in $\mathrm{MeOH}(10 \mathrm{~mL})$ and filtered with a PTFE membrane $(\phi 0.2 \mu \mathrm{m})$ using a disposable polyethylene syringe. The solution was concentrated under reduced pressure and the residue was digested with high-purity $70 \% \mathrm{HNO}_{3}(7 \mathrm{~mL})$ under microwave irradiation at $120-230{ }^{\circ} \mathrm{C}$. The resulting solution was diluted with ultrapure water to adjust the volume to $200 \mathrm{~mL}$ before analysis. The concentration of Pd was $0.19+/-0.05 \mathrm{ppb}$ in solution.

Multigram-scale reaction using the microflow device (Scheme 4). The mixture of aniline (1a, 50.0 mmol, $4.661 \mathrm{~g}$, purified by distillation), benzaldehyde (2a, $60.0 \mathrm{mmol}, 6.374 \mathrm{~g}$, purified by distillation), and tridecane (3.763 g; as an internal standard for GC analysis) in dehydrated 2-propanol (40 mL) was gently stirred (ca. $60 \mathrm{rpm}$ ) over molecular sieve (MS3A, $10.0 \mathrm{~g}$, activated prior to use) at room temperature for $18 \mathrm{~h}$ under Ar. The molecular sieves were filtered off and washed with dehydrated 2-propanol (ca. $100 \mathrm{~mL}$ ). The combined solution was diluted with dehydrated 2-propanol to adjust the volume to 2000 $\mathrm{mL}$ in a volumetric flask. The resulting solution (0.025 $\mathrm{M}$ based on 1a) was bubbled with Ar for $15 \mathrm{~min}$ (ca. $1000 \mathrm{~mL} / \mathrm{min}$ ), and then sonicated for $5 \mathrm{~min}$ in a glass bottle. The Ar bubbling and the sonication were repeated once more. The solution and $\mathrm{H}_{2}$ gas were fed to the catalyst housing equipped with SiNS$\operatorname{Pd}(h=25 \mu \mathrm{m}$, tandem connection $(2 \times))$ through a static mixer (Spica KC-M-S-GL (YMC Co., Ltd., Japan)), where the flow rate of the reactant solution was set to $0.650 \mathrm{~mL} / \mathrm{min}(0.975 \mathrm{mmol} / \mathrm{h})$ by a HPLC pump, and that of $\mathrm{H}_{2}$ gas was set to $9.0 \mathrm{~mL} / \mathrm{min}$ (at standard pressure and temperature; sccm) by a mass flow controller. The internal pressure was monitored at the HPLC pump to adjust the pressure to $0.6 \mathrm{MPa}$ (gauge pressure) using a backpressure valve. The reaction temperature was monitored by K-thermocouple at the aluminum bottom base of a catalyst housing $\left(60 \pm 1{ }^{\circ} \mathrm{C}\right)$. After GC analysis, the total 24 fractions (39.0 $\mathrm{mL}$ for $1 \mathrm{~h}$ ) were combined and concentrated under reduced pressure. The crude mixture was passed through a short plug of 3-aminopropyl-functionalyzed silica gel (12 g) using EtOAc as an eluent. The solution was concentrated under reduced pressure. The residue was purified by MPLC (silica gel, hexane/EtOAc as eluent) to obtain $N$-benzylaniline (3a) in $91 \%$ yield (3.90 g) for the $24-\mathrm{h}$ run. 


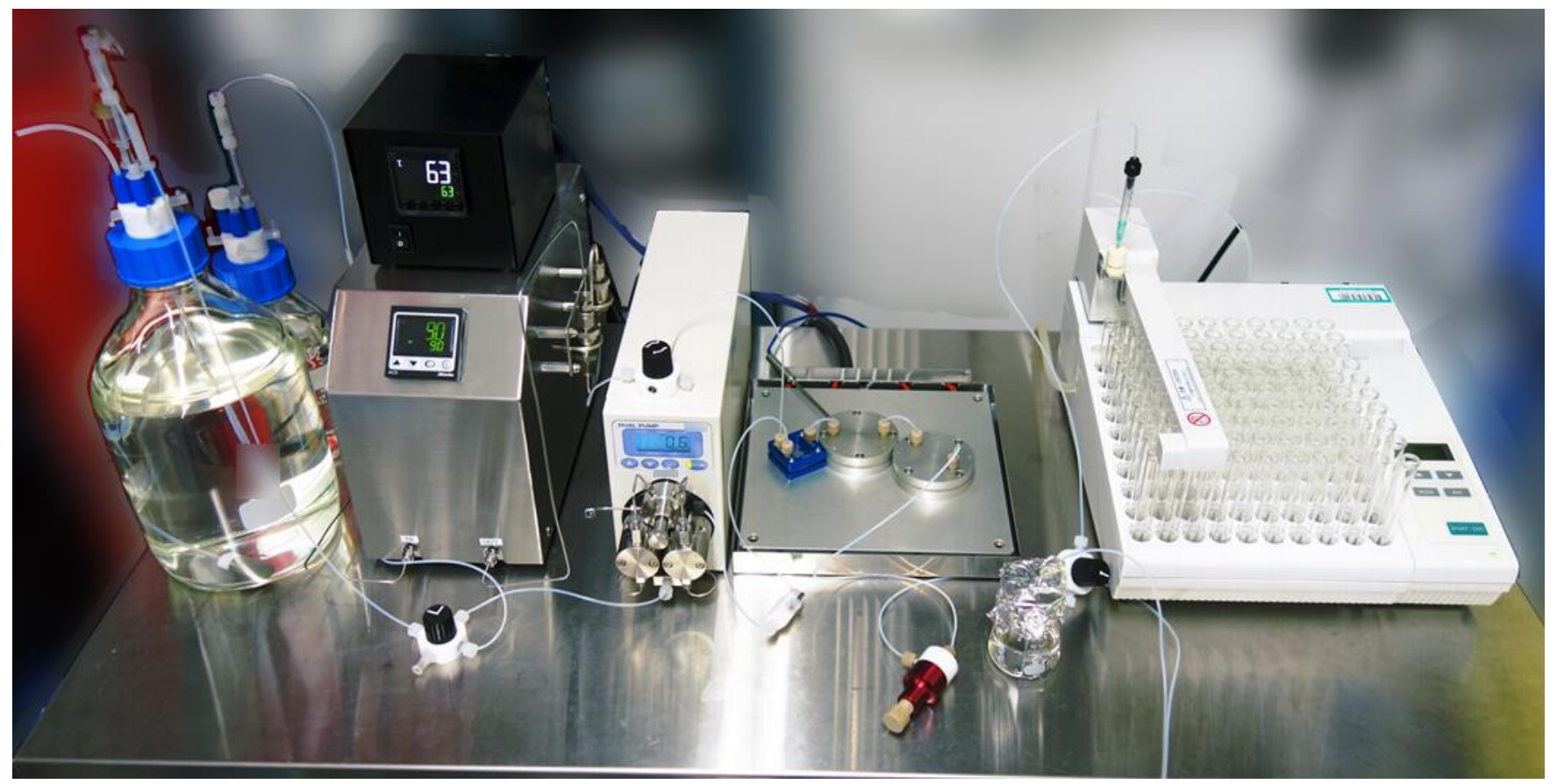

Figure S1. Appearance of the flow reaction system. Photograph courtesy of Takuma Sato. Copyright 2020 .

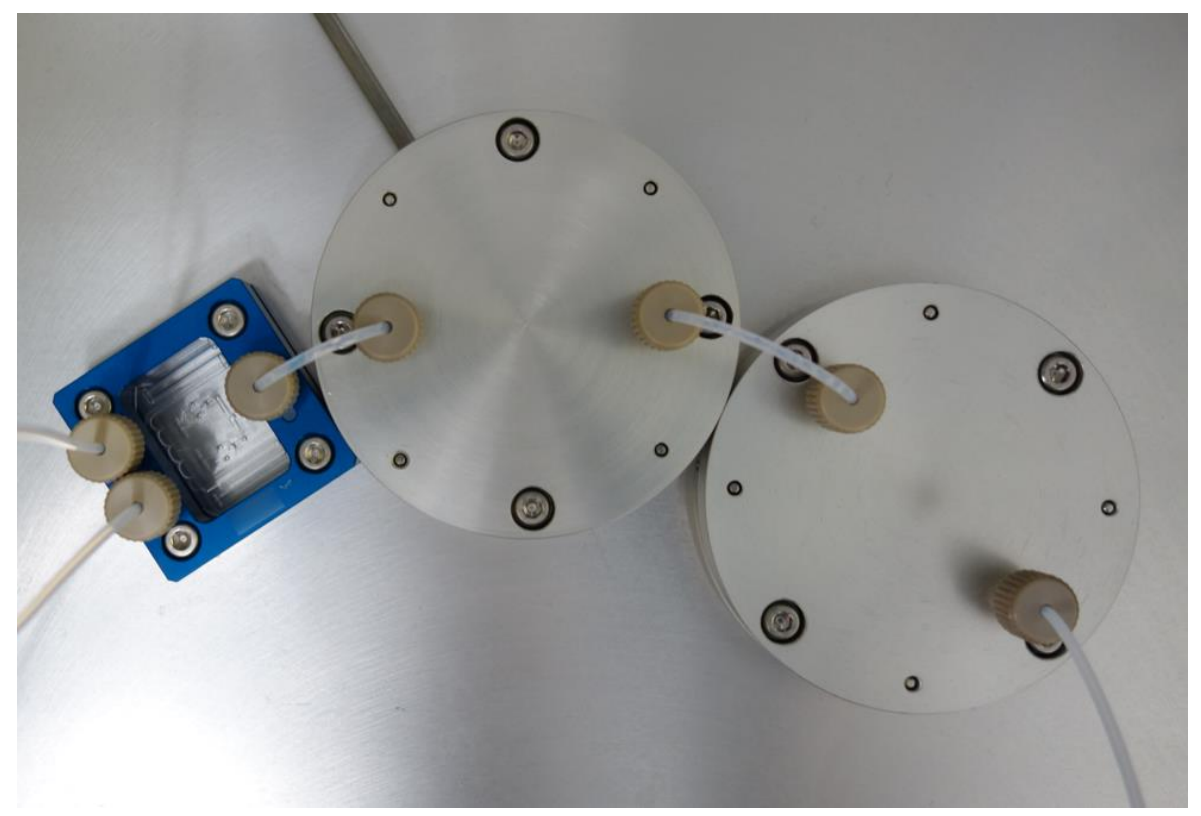

Figure S2. The microflow devices on a hotplate. Photograph courtesy of Takuma Sato. Copyright 2020. 
Scheme S1. Synthesis of (S)-2-(3,5-dimethylphenyl)pyrrolidine (1j)<smiles>CCOC(=O)C(C(=O)N(C)OC)C(C)C</smiles>

S1

S2<smiles>CC(C)(C)[SH](=O)=O</smiles><smiles>Cc1cc(C)cc([C@H]2CCCN2)c1</smiles>

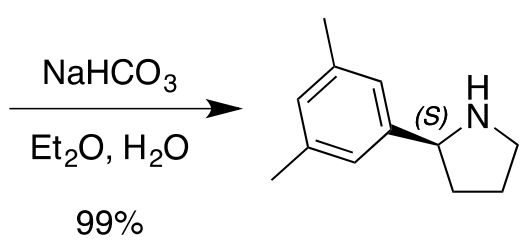

$1 \mathbf{j}$<smiles>CON(C)C(=O)CCCCl</smiles>

S1

$N$-Methoxy- $N$-methyl-4-chlorobutyramide (S1). To a mixture of $N, O$-dimethylhydroxylamine hydrochloride $(95.0 \mathrm{mmol}, 9.27 \mathrm{~g})$ in $\mathrm{CH}_{2} \mathrm{Cl}_{2}(150 \mathrm{~mL})$ was added $2 \mathrm{M} \mathrm{NaOH}$ (300 mmol, $150 \mathrm{~mL}$ ) and 4-chlorobutyryl chloride (100 mmol, $11.2 \mathrm{~mL}$ ) at $0{ }^{\circ} \mathrm{C}$. The mixture was stirred for $42 \mathrm{~h}$ at room temperature. The organic phase was separated, and the aqueous phase was extracted with $\mathrm{CH}_{2} \mathrm{Cl}_{2}(2 \times 50 \mathrm{~mL})$. The combined organic phase was washed with $2 \mathrm{M} \mathrm{NaOH}(100 \mathrm{~mL})$, dried over $\mathrm{Na}_{2} \mathrm{SO}_{4}$, filtered, and concentrated to afford the title comlund in $75 \%$ yield as a colorless liquid. ${ }^{1} \mathrm{H}$ NMR $\left(400 \mathrm{MHz}, \mathrm{CDCl}_{3}\right) \delta: 2.08-2.15$ $(\mathrm{m}, 2 \mathrm{H}), 2.63(\mathrm{t}, J=7.0 \mathrm{~Hz}, 2 \mathrm{H}), 3.19(\mathrm{~s}, 3 \mathrm{H}), 3.64(\mathrm{t}, J=6.3 \mathrm{~Hz}, 2 \mathrm{H}), 3.71(\mathrm{~s}, 3 \mathrm{H}) .{ }^{13} \mathrm{C}\left\{{ }^{1} \mathrm{H}\right\} \mathrm{NMR}(100$ $\left.\mathrm{MHz}_{2} \mathrm{CDCl}_{3}\right) \delta: 27.1,28.6,32.1,44.6,61.1$. IR $\left(v_{\max } / \mathrm{cm}^{-1}\right): 2965,2940,2821,1656,14421,1417,1387$, 1178, 1107, 997. HRMS (ESI+): calculated for [M+Na] $]^{+}: 188.0449$, found: 188.0450. 


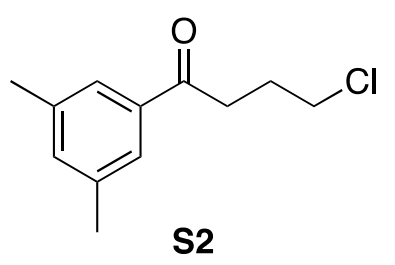

S2

4-Chloro-1-(3,5-dimethylphenyl)butan-1-one (S2). To a mixture of $N$-methoxy- $N$-methyl-4-chlorobutyramide (S1, $65.0 \mathrm{mmol}, 10.8 \mathrm{~g}$ ) in anhydrous $\mathrm{Et}_{2} \mathrm{O}$ $(100 \mathrm{~mL})$ was added dropwise 3,5-dimethylphenylmagnesium bromide (ca. $1 \mathrm{M}$ in $\mathrm{Et}_{2} \mathrm{O}$, ca. $130 \mathrm{mmol}$, prepared from 1-bromo-3,5-dimethylbenzene (130 mmol, $17.7 \mathrm{~mL})$ and $\mathrm{Mg}$ turnings $(169 \mathrm{mmol}, 4.11 \mathrm{~g})$ in anhydrous $\left.\mathrm{Et}_{2} \mathrm{O}(130 \mathrm{~mL})\right)$ over $1 \mathrm{~h}$ at $-40{ }^{\circ} \mathrm{C}$ under $\mathrm{Ar}$. The reaction mixture was stirred at room temperature for $20 \mathrm{~h}$. After cooling to $0{ }^{\circ} \mathrm{C}$, saturated $\mathrm{NH}_{4} \mathrm{Cl}$ solution $(200 \mathrm{~mL})$ was added. The organic phase was separated, washed with water $(100 \mathrm{~mL})$ and brine $(100 \mathrm{~mL})$, dried over $\mathrm{Na}_{2} \mathrm{SO}_{4}$, and filtered. After concentration, the residue was purified by column chromatography (silica gel, hexane/EtOAc as eluent) to afford the title compound in $91 \%$ yield as a greenish yellow liquid. ${ }^{1} \mathrm{H}$ NMR (400 MHz, $\left.\mathrm{CDCl}_{3}\right) \delta: 2.18-2.25(\mathrm{~m}, 2 \mathrm{H}), 2.38(\mathrm{~s}, 6 \mathrm{H}), 3.15(\mathrm{t}, J=7.0 \mathrm{~Hz}, 2 \mathrm{H})$, $3.67(\mathrm{t}, J=6.3 \mathrm{~Hz}, 2 \mathrm{H}), 7.21(\mathrm{~s}, 1 \mathrm{H}), 7.58(\mathrm{~s}, 2 \mathrm{H}) .{ }^{13} \mathrm{C}\left\{{ }^{1} \mathrm{H}\right\} \mathrm{NMR}\left(100 \mathrm{MHz}, \mathrm{CDCl}_{3}\right) \delta: 21.2,26.8,35.4$, 44.7, 125.8, 134.8, 136.8, 138.3, 199.4. IR $\left(v_{\max } / \mathrm{cm}^{-1}\right): 3047,3006,2961,2920,2868,1443,1411,1322$, 1303, 1181, 1159, 844, 785, 687. HRMS (APCI+): calculated for [M+H] $]^{+}: 211.0884$, found: 211.0884.

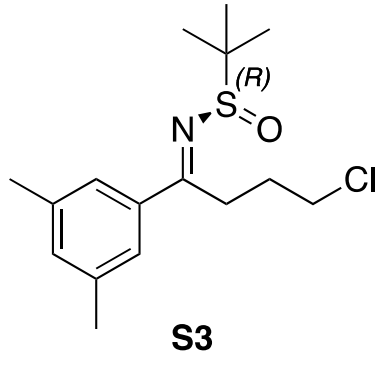

$300 \mathrm{~mL}$ ). The organic was separated, dried over $\mathrm{Na}_{2} \mathrm{SO}_{4}$, and filtered. After concentration under reduced pressure, the residue was purified by column chromatography (silica gel, hexane/EtOAc as eluent) to afford the title compound in $57 \%$ yield as a brown viscous liquid. ${ }^{1} \mathrm{H} \mathrm{NMR}\left(400 \mathrm{MHz}, \mathrm{CDCl}_{3}\right) \delta: 1.33$ (s, 9H), 2.10-2.22 (m, 2H), $2.36(\mathrm{~s}, 6 \mathrm{H}), 3.27(\mathrm{~s}, 1 \mathrm{H}), 3.43(\mathrm{~s}, 1 \mathrm{H}), 3.64(\mathrm{t}, J=6.5 \mathrm{~Hz}, 2 \mathrm{H}), 7.13(\mathrm{~s}, 1 \mathrm{H})$, 7.47 (s, 2H). ${ }^{13} \mathrm{C}\left\{{ }^{1} \mathrm{H}\right\}$ NMR $\left(100 \mathrm{MHz}, \mathrm{CDCl}_{3}\right) \delta: 21.3,22.7,30.2,31.6,44.7,57.7,125.2,133.4,137.6$, 138.2, 178.6. IR $\left(v_{\max } / \mathrm{cm}^{-1}\right): 3046,2958,2922,2866,1599,1577,1455,1361,1320,1308,1069,856$. HRMS (ESI+): calculated for $[\mathrm{M}+\mathrm{H}]^{+}: 314.1340$, found: $314.1344 .[\alpha]_{\mathrm{D}}^{20}+11.0\left(c=1.01, \mathrm{CH}_{2} \mathrm{Cl}_{2}\right)$. 


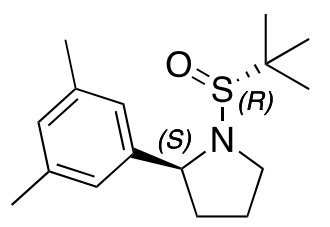

$\mathbf{S 4}$

$\left(R_{\mathrm{S}}, S\right)$-1-tert-Butylsulfinyl-2-(3,5-dimethylphenyl)pyrrolidine (S4). To a solution of $\left(R_{\mathrm{S}}\right)-N$-(4-chloro-1-(3,5-dimethylphenyl)butylidene)-tert-butanesulfinamide $(\mathbf{S 3}, 25.6 \mathrm{mmol}, 8.06 \mathrm{~g})$ in anhydrous THF $(100 \mathrm{~mL})$ at $-78{ }^{\circ} \mathrm{C}$ was added $\mathrm{LiBEt}_{3} \mathrm{H}$ (28 mmol, 0.5 M in THF, $28.2 \mathrm{~mL}$ ) under Ar. The reaction was stirred at $-78{ }^{\circ} \mathrm{C}$ for $1 \mathrm{~h}$, subsequently allowed to warm up to room temperature and stirred for additional 20 h. Saturated $\mathrm{NaHCO}_{3}$ solution $(80 \mathrm{~mL})$ was slowly added. The mixture was filtered and extracted with EtOAc $(3 \times 100 \mathrm{~mL})$. The combined organic phase was dried over $\mathrm{Na}_{2} \mathrm{SO}_{4}$ and filtered. After concentration, the residue was purified by column chromatography (silica gel, hexane/EtOAc as eluent) to afford the title compound in $72 \%$ yield as pale yellow solid. mp.: $56{ }^{\circ} \mathrm{C}$. ${ }^{1} \mathrm{H} \mathrm{NMR}\left(400 \mathrm{MHz}, \mathrm{CDCl}_{3}\right)$ $\delta: 1.12(\mathrm{~s}, 9 \mathrm{H}), 1.74-1.90(\mathrm{~m}, 3 \mathrm{H}), 1.93-2.02(\mathrm{~m}, 1 \mathrm{H}), 2.18-2.27(\mathrm{~m}, 1 \mathrm{H}), 2.30(\mathrm{~s}, 6 \mathrm{H}), 2.94-3.02(\mathrm{~m}, 1 \mathrm{H})$, 3.85-3.91 (m, 1H), 4.55-4.59 (m, 1H), $6.88(\mathrm{~s}, 1 \mathrm{H}), 6.90(\mathrm{~s}, 2 \mathrm{H}) .{ }^{13} \mathrm{C}\left\{{ }^{1} \mathrm{H}\right\} \mathrm{NMR}\left(100 \mathrm{MHz}, \mathrm{CDCl}_{3}\right) \delta$ : 21.3, 23.8, 26.3, 36.0, 42.1, 57.2, 69.2, 125.0, 128.7, 137.7, 143.2. IR $\left(v_{\max } / \mathrm{cm}^{-1}\right)$ : 3023, 2957, 2920, 2866, 1607, 1471, 1360, 1061, 957, 847. HRMS (ESI+): calculated for $[\mathrm{M}+\mathrm{Na}]^{+}:$302.1549, found: 302.1548. $[\alpha]_{\mathrm{D}}^{20}-137\left(c=0.49, \mathrm{CH}_{2} \mathrm{Cl}_{2}\right)$.

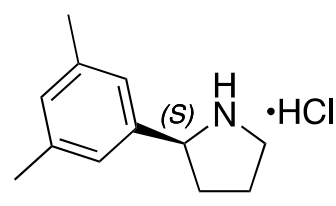

$1 \mathrm{j} \cdot \mathrm{HCl}$

(S)-2-(3,5-Dimethylphenyl)pyrrolidine hydrochloride $(\mathbf{1 j} \cdot \mathbf{H C l})$. To a solution of $\left(R_{\mathrm{S}}, S\right)$-1-tert-butylsulfinyl-2-(3,5-dimethylphenyl)pyrrolidine (S4, $14.7 \mathrm{mmol}$, $4.12 \mathrm{~g}$ ) in dioxane $(250 \mathrm{~mL})$ was added dropwise $\mathrm{HCl}$ (ca. $150 \mathrm{mmol}, 4 \mathrm{M}$ in dioxane, $38 \mathrm{~mL}$ ). The mixture was stirred for $1 \mathrm{~h}$ at room temperature under $\mathrm{N}_{2}$, and then the mixture was concentrated under reduced pressure. Then, $\mathrm{Et}_{2} \mathrm{O}(200 \mathrm{~mL})$ was added to the residue and the mixture was cooled to $0{ }^{\circ} \mathrm{C}$. The precipitate was collected by filtration, washed with $\mathrm{Et}_{2} \mathrm{O}(40$ $\mathrm{mL}$ ), and dried under reduced pressure to afford the title compound in 94\% yield as white solid. mp.: 198 ${ }^{\circ} \mathrm{C}$. ${ }^{1} \mathrm{H}$ NMR $\left(400 \mathrm{MHz}, \mathrm{D}_{2} \mathrm{O}\right) \delta:$ 2.00-2.15 (m, 3H), $2.18(\mathrm{~s}, 6 \mathrm{H}), 2.27-2.35(\mathrm{~m}, 1 \mathrm{H}), 3.27-3.36(\mathrm{~m}, 2 \mathrm{H})$, $4.45(\mathrm{t}, J=8.0 \mathrm{~Hz}, 1 \mathrm{H}), 6.97(\mathrm{~s}, 2 \mathrm{H}), 7.01(\mathrm{~s}, 1 \mathrm{H}) .{ }^{13} \mathrm{C}\left\{{ }^{1} \mathrm{H}\right\} \mathrm{NMR}\left(100 \mathrm{MHz}, \mathrm{D}_{2} \mathrm{O}\right) \delta: 20.9,24.19,30.9$, 46.0, 63.8, 119.79, 125.6, 131.4, 135.3, 140.1. IR $\left(v_{\max } / \mathrm{cm}^{-1}\right): 3033,3012,2970,2855,2743,2571,2480$, 1608, 1590, 1414, 850. HRMS (ESI+): calculated for $[\mathrm{M}-\mathrm{Cl}]^{+}: 176.1434$, found: $176.1435 .[\alpha]_{\mathrm{D}}{ }^{20}+7.1$ $(c=1.01, \mathrm{MeOH})$. 


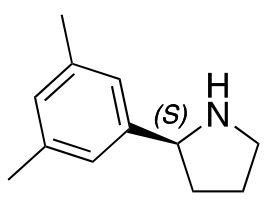

1j

(S)-2-(3,5-Dimethylphenyl)pyrrolidine (1j). To a suspension of $(S)$-2-(3,5-dimethylphenyl)pyrrolidine hydrochloride $(\mathbf{1} \mathbf{j} \cdot \mathrm{HCl}, 13.5 \mathrm{mmol}, 2.86 \mathrm{~g})$ in anhydrous $\mathrm{Et}_{2} \mathrm{O}$ $(200 \mathrm{~mL})$ was added a saturated solution of $\mathrm{NaHCO}_{3}(200 \mathrm{~mL})$. The resulting mixture was stirred for $20 \mathrm{~min}$ at room temperature. The organic was separated and the aqueous phase was extracted with $\mathrm{Et}_{2} \mathrm{O}(2 \times 100 \mathrm{~mL})$. The combined organic phase was dried over $\mathrm{MgSO}_{4}$ and filtered. The solvent was removed under reduced pressure to afford the title compound as a pale yellow liquid in 99\% yield. ${ }^{1} \mathrm{H}$ NMR $\left(400 \mathrm{MHz}, \mathrm{CDCl}_{3}\right) \delta: 1.60-1.71(\mathrm{~m}, 1 \mathrm{H}), 1.78-1.96(\mathrm{~m}, 2 \mathrm{H}), 1.98(\mathrm{~s}, 1 \mathrm{H})$, 2.11-2.19 (m, 1H), $2.30(\mathrm{~s}, 6 \mathrm{H}), 2.95-3.02(\mathrm{~m}, 1 \mathrm{H}), 3.17-3.23(\mathrm{~m}, 1 \mathrm{H}), 4.03(\mathrm{t}, J=7.7 \mathrm{~Hz}, 1 \mathrm{H}), 6.87(\mathrm{~s}$, 1H), 6.97 (s, 2H). $\left.{ }^{13} \mathrm{C}^{1}{ }^{1} \mathrm{H}\right\}$ NMR $\left(100 \mathrm{MHz}, \mathrm{CDCl}_{3}\right) \delta: 21.3,25.5,34.2,46.9,62.6,124.2,128.4,137.8$, 144.7. IR $\left(v_{\max } / \mathrm{cm}^{-1}\right): 3332,3010,2960,2915,2869,1605,1458,1101,845$. HRMS (ESI+): calculated for $[\mathrm{M}+\mathrm{H}]^{+}:$176.1434, found: $176.1436 .[\alpha]_{\mathrm{D}}^{20}-30.5(c=1.01, \mathrm{MeOH})$. Chiral HPLC (ChiralPak OD$\mathrm{H}, \phi 4.6 \mathrm{~mm} \times \mathrm{L} 250 \mathrm{~mm}$, hexane:2-propanol $=90: 10,0.5 \mathrm{~mL} / \mathrm{min}, \lambda=254 \mathrm{~nm}): t_{\mathrm{R}} / \mathrm{min}=18.7(1 \%)$, $19.8(99 \%)$.

Determination of the enantiomeric ratio of $\mathbf{1} \mathbf{j}$. Before chiral HPLC analysis, $\mathbf{1} \mathbf{j}$ was derivatized with benzoyl chloride as follows. To a solution of a small amount of $\mathbf{1} \mathbf{j}$ (ca. $10 \mathrm{mg}$ ) in $\mathrm{CH}_{2} \mathrm{Cl}_{2}(2.5 \mathrm{~mL}$ ) was added $2 \mathrm{M} \mathrm{NaOH}(0.50 \mathrm{mmol}, 2.5 \mathrm{~mL})$ and benzoyl chloride $(0.20 \mathrm{mmol}, 23 \mu \mathrm{L})$ in a glass tube. The mixture was agitated using a vortex mixer for $4 \mathrm{~h}$ at room temperature. The separated organic phase was treated with $2 \mathrm{M} \mathrm{NaOH}$ (ca. $2 \mathrm{~mL}$ ) and the resulting organic solution was passed through a small column of $\mathrm{Na}_{2} \mathrm{SO}_{4}$. After removal of volatile materials under reduced pressure, the residue was diluted with HPLC grade hexane and passed though a PTFE membrane filter $(\phi 0.2 \mu \mathrm{m})$, which was subjected to chiral HPLC analysis without further purification. Racemic mixture of $\mathbf{1} \mathbf{j}$ was also analyzed in a similar way. 
<smiles>O=Cc1ccc(O)cc1</smiles><smiles>N#Cc1ccc(F)c(F)c1</smiles><smiles>NC(=O)c1ccc(Oc2ccc(C=O)cc2)c(F)c1</smiles>

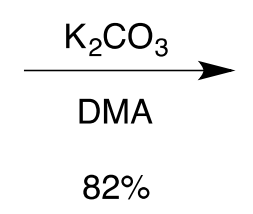

$82 \%$<smiles>N#Cc1ccc(Oc2ccc(C=O)cc2)c(F)c1</smiles>

S5

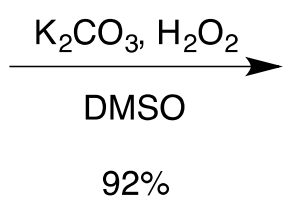

$92 \%$<smiles>N#Cc1ccc(Oc2ccc(C=O)cc2)c(F)c1</smiles>

S5

3-Fluoro-4-(4-formylphenoxy)benzonitrile ${ }^{2}$ (S5). A mixture of 3,4difluorobenzonitrile (35.0 mmol, $4.87 \mathrm{~g})$, 4-hydroxybenzaldehyde (35.0 mmol, $4.27 \mathrm{~g}$ ), and $\mathrm{K}_{2} \mathrm{CO}_{3}(70.0 \mathrm{mmol}, 9.67 \mathrm{~g})$ in $N, N$-dimethylacetamide $(90 \mathrm{~mL})$ was stirred at $100{ }^{\circ} \mathrm{C}$ for $2 \mathrm{~h}$ under $\mathrm{N}_{2}$. After cooling, the reaction mixture was poured into ice water. White precipitate was collected by filtration, washed with water, and dried under reduced pressure to afford the title compound as pale yellow solid in $82 \%$ yield. mp.: $101{ }^{\circ} \mathrm{C} .{ }^{1} \mathrm{H}$ NMR $\left(400 \mathrm{MHz} \mathrm{CDCl}_{3}\right) \delta: 7.11-7.15(\mathrm{~m}, 2 \mathrm{H}), 7.20(\mathrm{t}, J=8.2 \mathrm{~Hz}$, 1H), 7.49-7.51 (m, 1H), $7.54(\mathrm{dd}, J=9.7,1.9 \mathrm{~Hz}, 1 \mathrm{H}), 7.91-7.94(\mathrm{~m}, 2 \mathrm{H}), 9.98(\mathrm{~s}, 1 \mathrm{H}) .{ }^{13} \mathrm{C}\left\{{ }^{1} \mathrm{H}\right\} \mathrm{NMR}$ $\left(100 \mathrm{MHz}, \mathrm{CDCl}_{3}\right) \delta: 109.1\left(\mathrm{~d},{ }^{3} \mathrm{~J}_{\mathrm{C}-\mathrm{F}}=8.2 \mathrm{~Hz}\right), 117.1\left(\mathrm{~d},{ }^{4} J_{\mathrm{C}-\mathrm{F}}=2.5 \mathrm{~Hz}\right), 117.9,121.3\left(\mathrm{~d},{ }^{2} J_{\mathrm{C}-\mathrm{F}}=21.3 \mathrm{~Hz}\right)$, $122.5\left(\mathrm{~d},{ }^{4} J_{\mathrm{C}-\mathrm{F}}=1.6 \mathrm{~Hz}\right), 129.6\left(\mathrm{~d},{ }^{3} J_{\mathrm{C}-\mathrm{F}}=4.1 \mathrm{~Hz}\right), 132.1,132.7,147.0\left(\mathrm{~d},{ }^{2} J_{\mathrm{C}-\mathrm{F}}=11.5 \mathrm{~Hz}\right), 153.6\left(\mathrm{~d},{ }^{1} J_{\mathrm{C}-}\right.$ $\mathrm{F}=254.8 \mathrm{~Hz}), 160.7,190.4 . \mathrm{IR}\left(v_{\max } / \mathrm{cm}^{-1}\right): 3100,3060,2846,2812,2761,2232,1697,1687,1585,1497$, 1277, 1216, 1166, 1114, 836. HRMS (APCI+): calculated for [M+H] $]^{+}: 242.0612$, found: 242.0616.<smiles>NC(=O)c1ccc(Oc2ccc(C=O)cc2)c(F)c1</smiles>

3-Fluoro-4-(4-formylphenoxy)benzamide ${ }^{2}$ (2f). To a mixture of 3fluoro-4-(4-formylphenoxy)benzonitrile (S5, $26.0 \mathrm{mmol}, 6.27 \mathrm{~g}$ ) and $\mathrm{K}_{2} \mathrm{CO}_{3}(13.0 \mathrm{mmol}, 1.80 \mathrm{~g})$ in DMSO $(24 \mathrm{~mL})$ was added dropwise $35 \%$ $\mathrm{H}_{2} \mathrm{O}_{2}$ (ca. $29 \mathrm{mmol}, 3.1 \mathrm{~mL}$ ) at $10{ }^{\circ} \mathrm{C}$ over $5 \mathrm{~min}$. The reaction mixture was stirred at room temperature for $2 \mathrm{~h}$. The reaction mixture was poured into ice water. White precipitate was collected by filtration, washed with water, and dried under reduced pressure to afford the title compound as white solid in $92 \%$ yield. mp. $129{ }^{\circ} \mathrm{C}$. ${ }^{1} \mathrm{H}$ NMR $(400$ $\left.\mathrm{MHz},\left(\mathrm{D}_{3} \mathrm{C}\right)_{2} \mathrm{SO}\right) \delta: 9.96(\mathrm{~s}, 1 \mathrm{H}), 8.12(\mathrm{~s}, 1 \mathrm{H}), 7.96(\mathrm{~d}, J=8.2 \mathrm{~Hz}, 2 \mathrm{H}), 7.93(\mathrm{dd}, J=1.9,10.0 \mathrm{~Hz}, 1 \mathrm{H})$, 
7.85-7.82 (m, 1H), $7.58(\mathrm{~s}, 1 \mathrm{H}), 7.42(\mathrm{t}, J=8.2 \mathrm{~Hz}, 1 \mathrm{H}), 7.20(\mathrm{~d}, J=8.2 \mathrm{~Hz}, 2 \mathrm{H}) .{ }^{13} \mathrm{C}\left\{{ }^{1} \mathrm{H}\right\} \mathrm{NMR}(100$ $\left.\mathrm{MHz},\left(\mathrm{D}_{3} \mathrm{C}\right)_{2} \mathrm{SO}\right) \delta: 116.6\left(\mathrm{~d},{ }^{2} J_{\mathrm{C}-\mathrm{F}}=19.7 \mathrm{~Hz}\right), 116.9,122.6,125.1\left(\mathrm{~d},{ }^{4} J_{\mathrm{C}-\mathrm{F}}=3.3 \mathrm{~Hz}\right), 131.9\left(\mathrm{~d},{ }^{2} J_{\mathrm{C}-\mathrm{F}}=\right.$ 21.3 Hz), 132.1, $132.7\left(\mathrm{~d},{ }^{3} J_{\mathrm{C}-\mathrm{F}}=5.7 \mathrm{~Hz}\right), 143.7\left(\mathrm{~d},{ }^{3} J_{\mathrm{C}-\mathrm{F}}=12.3 \mathrm{~Hz}\right), 153.1\left(\mathrm{~d},{ }^{1} J_{\mathrm{C}-\mathrm{F}}=248.2 \mathrm{~Hz}\right), 161.3$, 165.8, 191.5. IR ( $\left.v_{\max } / \mathrm{cm}^{-1}\right): 3356,3185,2844,1668,1598,1504,1433,1382,1269,1218,1156,1128$, 830. HRMS (ESI+): calculated for $[\mathrm{M}+\mathrm{Na}]^{+}:$282.0537, found: 282.0541. HRMS (APCI+): calculated for $[\mathrm{M}+\mathrm{H}]^{+}:$260.0717, found: 260.0716 .

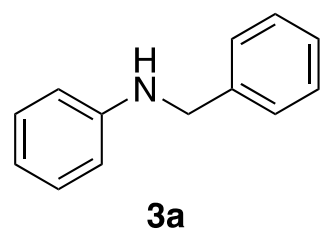

N-Benzylaniline (3a): White solid. mp.: $37{ }^{\circ} \mathrm{C}$. ${ }^{1} \mathrm{H}$ NMR $\left(400 \mathrm{MHz}, \mathrm{CDCl}_{3}\right) \delta$ : 4.01 (br s, 1H), 4.32 (s, 2H), 6.61-6.64 (m, 2H), 6.71 (tt, $J=7.2,1.0 \mathrm{~Hz}, 1 \mathrm{H}), 7.15$ $7.19(\mathrm{~m}, 2 \mathrm{H}), 7.25-7.28(\mathrm{~m}, 1 \mathrm{H}), 7.33-7.36(\mathrm{~m}, 4 \mathrm{H}) .{ }^{13} \mathrm{C}\left\{{ }^{1} \mathrm{H}\right\}$ NMR $(100 \mathrm{MHz}$, $\left.\mathrm{CDCl}_{3}\right) \delta: 48.3,112.8,117.5,127.2,127.9,128.6,129.2,139.4,148.1 . \mathrm{IR}\left(v_{\max } / \mathrm{cm}^{-}\right.$ $\left.{ }^{1}\right): 3417,3080,3052,3023,2925,2846,1600,1508,1492,1448,1432,1328,1277,1179,984,856,734$, 687. HRMS (ESI+): calculated for $[\mathrm{M}+\mathrm{H}]^{+}:$184.1121, found: 184.1124 .

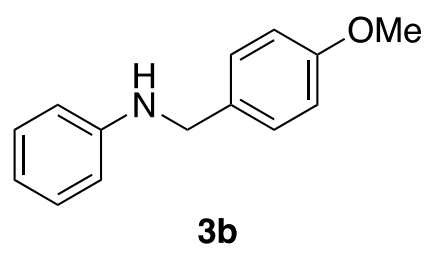

$N$-(4-Methoxybenzyl)aniline (3b): White solid. mp.: $47{ }^{\circ} \mathrm{C} .{ }^{1} \mathrm{H}$ NMR (400 $\left.\mathrm{MHz}, \mathrm{CDCl}_{3}\right) \delta: 3.80$ (s, 3H), 3.94 (br s, 1H), 4.25 (s, 2H), 6.62-6.65 (m, 2H), 6.69-6.73 (m, 1H), 6.86-6.90 (m, 2H), 7.15-7.19 (m, 2H), 7.29 (d, J=8.2 Hz, $2 \mathrm{H}) .{ }^{13} \mathrm{C}\left\{{ }^{1} \mathrm{H}\right\} \mathrm{NMR}\left(100 \mathrm{MHz}, \mathrm{CDCl}_{3}\right) \delta: 47.8,55.3,112.8,114.0,117.5$, 128.8, 129.2 131.4, 148.2, 158.8. IR ( $\left.v_{\max } / \mathrm{cm}^{-1}\right): 3397,3077,3049,3022,3004,2962,2934,2897,2836$, 1601, 1582, 1511, 1499, 1301, 1246, 1171, 1033, 746, 692. HRMS (ESI+): calculated for [M+H] 214.1226, found: 214.1228 .

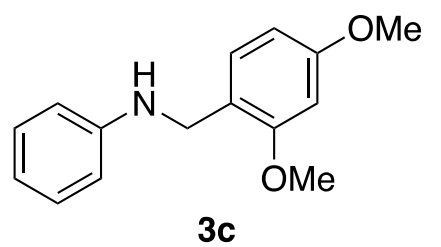

$N$-(2,4-Dimethoxybenzyl)aniline (3c): White solid. mp.: $99{ }^{\circ} \mathrm{C}$. ${ }^{1} \mathrm{H}$ NMR $\left(400 \mathrm{MHz}, \mathrm{CDCl}_{3}\right) \delta: 3.79(\mathrm{~s}, 3 \mathrm{H}), 3.82(\mathrm{~s}, 3 \mathrm{H}), 4.24(\mathrm{~s}, 2 \mathrm{H}), 6.42(\mathrm{dd}, J=$ 8.2, $2.4 \mathrm{~Hz}, 1 \mathrm{H}), 6.47$ (d, $J=2.4 \mathrm{~Hz}, 1 \mathrm{H}), 6.64-6.66(\mathrm{~m}, 2 \mathrm{H}), 6.66-6.70$ (m, $1 \mathrm{H}), 7.13-7.18(\mathrm{~m}, 2 \mathrm{H}), 7.19(\mathrm{~d}, J=8.2 \mathrm{~Hz}, 1 \mathrm{H}) .{ }^{13} \mathrm{C}\left\{{ }^{1} \mathrm{H}\right\} \mathrm{NMR}(100 \mathrm{MHz}$, $\left.\mathrm{CDCl}_{3}\right) \delta: 43.2,55.3,55.4,98.6,103.8,113.1,117.3,119.7,129.1,129.7,148.4,158.4,160.2$. IR $\left(v_{\max } / \mathrm{cm}^{-1}\right): 3372,3083,3052,2997,2965,2941,2915,2874,2836,1602,1506,1468,1434,1320,1254$, 1207, 1154, 1130, 922, 831, 748. HRMS (ESI+): calculated for [M+H] $]^{+}: 244.1332$, found: 244.1333. 


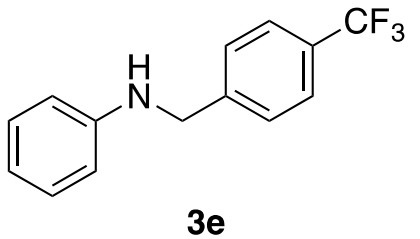

$3 e$

$N$-(4-(Trifluoromethyl)benzyl)aniline (3d): Colorless liquid. ${ }^{1} \mathrm{H}$ NMR (400 $\left.\mathrm{MHz}, \mathrm{CDCl}_{3}\right) \delta: 4.12$ (br s, 1H), 4.39 (s, 2H), 6.57-6.61 (m, 2H), 6.70-6.75 (m, 1H), 7.14-7.19 (m, 2H), $7.46(\mathrm{~d}, J=8.2 \mathrm{~Hz}, 2 \mathrm{H}), 7.58(\mathrm{~d}, J=8.2 \mathrm{~Hz}, 2 \mathrm{H})$. ${ }^{13} \mathrm{C}\left\{{ }^{1} \mathrm{H}\right\} \mathrm{NMR}\left(100 \mathrm{MHz}, \mathrm{CDCl}_{3}\right) \delta: 47.7,112.9,117.9,124.2\left(\mathrm{q},{ }^{1} J_{\mathrm{C}-\mathrm{F}}=272.0\right.$ $\mathrm{Hz}), 125.52\left(\mathrm{q},{ }^{3} J_{\mathrm{C}-\mathrm{F}}=3.8 \mathrm{~Hz}\right), 127.4129 .3,129.4\left(\mathrm{q},{ }^{2} J_{\mathrm{C}-\mathrm{F}}=32.0 \mathrm{~Hz}\right), 143.7,147.6 . \mathrm{IR}\left(v_{\max } / \mathrm{cm}^{-1}\right): 3421$, 3054, 3022, 2922, 2846, 1603, 1506, 1418, 1321, 1161, 1111, 1065, 1017, 827, 748, 691. HRMS (ESI+): calculated for $[\mathrm{M}+\mathrm{H}]^{+}:$252.0995, found: 252.0996.

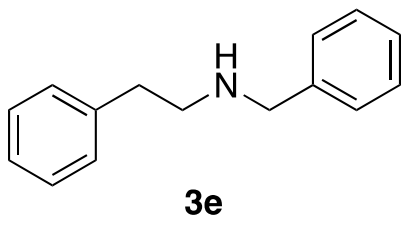

$N$-Benzyl-2-phenylethylamine (3e): Colorless liquid. ${ }^{1} \mathrm{H}$ NMR (400 MHz, $\left.\mathrm{CDCl}_{3}\right) \delta: 1.54($ br s, $1 \mathrm{H}), 2.80-2.84(\mathrm{~m}, 2 \mathrm{H}), 2.89-2.92(\mathrm{~m}, 2 \mathrm{H}), 3.79(\mathrm{~s}, 2 \mathrm{H})$, 7.18-7.32 (m, 10H). ${ }^{13} \mathrm{C}\left\{{ }^{1} \mathrm{H}\right\}$ NMR $\left(100 \mathrm{MHz}, \mathrm{CDCl}_{3}\right) \delta: 36.3,50.5,53.8$, $126.1,126.9,128.0,128.3,128.4,128.7,140.0,140.2$. IR $\left(v_{\max } / \mathrm{cm}^{-1}\right): 3309$, 3084, 3061, 3026, 2923, 2817, 1603, 1495, 1453, 1117, 1029, 734, 695. HRMS (ESI+): calculated for $[\mathrm{M}+\mathrm{H}]^{+}:$212.1434, found: 212.1434 .

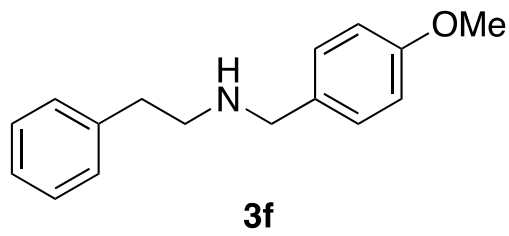

$N$-(4-Methoxybenzyl)-2-phenylethylamine (3f): Colorless liquid. ${ }^{1} \mathrm{H}$ NMR (400 MHz, $\left.\mathrm{CDCl}_{3}\right) \delta: 1.55$ (br s, 1H), 2.79-2.83 (m, 2H), 2.86$2.91(\mathrm{~m}, 2 \mathrm{H}), 3.73(\mathrm{~s}, 2 \mathrm{H}), 3.78(\mathrm{~s}, 3 \mathrm{H}), 6.83-6.85(\mathrm{~m}, 2 \mathrm{H}), 7.18-7.20$

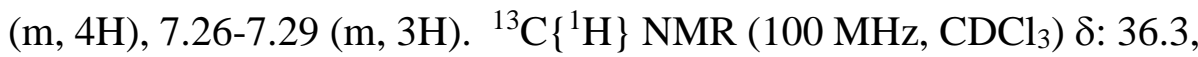
50.4, 53.2, 55.2, $113.7,126.1,128.4,128.7,129.2,132.4,140.0,158.5 . \quad$ IR $\left(v_{\max } / \mathrm{cm}^{-1}\right): 3313,3061,3026$, 3001, 2932, 2833, 1610, 1510, 1454, 1243, 1173, 1034, 744, 698. HRMS (ESI+): calculated for [M+H] $]^{+}$ 242.1539, found: 242.1538 .

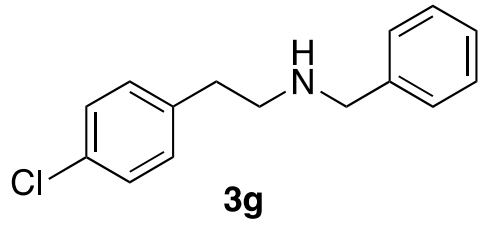

$N$-Benzyl-2-(4-chlorophenyl)ethylamine (3g): Colorless liquid. $\quad{ }^{1} \mathrm{H}$ NMR (400 MHz, $\left.\mathrm{CDCl}_{3}\right) \delta: 1.50($ br s, $1 \mathrm{H}), 2.76-2.80(\mathrm{~m}, 2 \mathrm{H}), 2.85-2.89$ $(\mathrm{m}, 2 \mathrm{H}), 3.79(\mathrm{~s}, 2 \mathrm{H}), 7.11-7.13(\mathrm{~m}, 2 \mathrm{H}), 7.22-7.33(\mathrm{~m}, 7 \mathrm{H}) .{ }^{13} \mathrm{C}\left\{{ }^{1} \mathrm{H}\right\}$ NMR (100 MHz, $\left.\mathrm{CDCl}_{3}\right) \delta: 35.7,50.3,53.8,126.9,128.0,128.4,128.5$, 130.0, 131.9, 138.5, 140.1. IR $\left(v_{\max } / \mathrm{cm}^{-1}\right): 3323,3085,3062,3027,2925,2817,1492,1454,1107,1090$, 1014, 807, 733, 697. HRMS (ESI+): calculated for $[\mathrm{M}+\mathrm{H}]^{+}:$246.1044, found: 246.1046. 


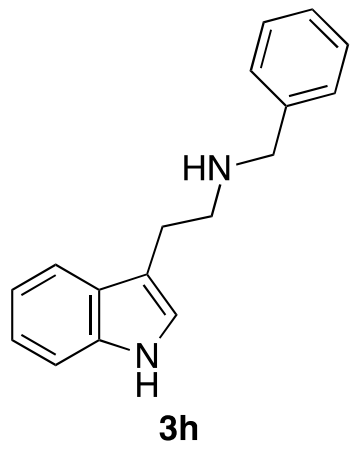

$N$-Benzyltryptamine (3h): Yellow liquid. ${ }^{1} \mathrm{H}$ NMR $\left(400 \mathrm{MHz}, \mathrm{CDCl}_{3}\right) \delta: 1.65$ (br s, 1H), 2.99 (s, 4H), 3.80 (s, 2H), $6.95(\mathrm{~d}, J=1.9 \mathrm{~Hz}, 1 \mathrm{H}), 7.09-7.11(\mathrm{~m}, 1 \mathrm{H})$, 7.16-7.32 (m, 7H), $7.60(\mathrm{~d}, J=7.7 \mathrm{~Hz}, 1 \mathrm{H}), 8.19(\mathrm{~s}, 1 \mathrm{H}) .{ }^{13} \mathrm{C}\left\{{ }^{1} \mathrm{H}\right\}$ NMR $(100$ $\left.\mathrm{MHz}, \mathrm{CDCl}_{3}\right) \delta: 25.7,49.3,53.8,111.1,113.8,118.9,119.2,121.9,121.9,126.8$, 127.4, 128.1, 128.3, 136.4, 140.3. IR ( $\left.v_{\max } / \mathrm{cm}^{-1}\right): 3413,3142,3058,2919,2841$, 1495, 1455, 1354, 1340, 1228, 1101, 736, 697. HRMS (ESI+): calculated for $[\mathrm{M}+\mathrm{H}]^{+}:$251.1543, found: 251.1542 .

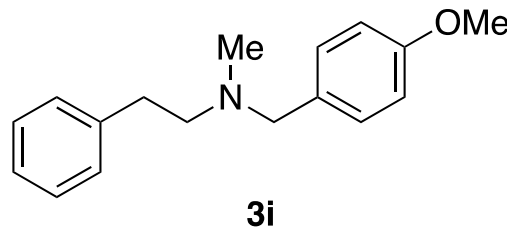

$N$-(4-Methoxybenzyl)- $N$-methyl-2-phenylethylamine (3i): Colorless liquid. ${ }^{1} \mathrm{H} \mathrm{NMR}\left(400 \mathrm{MHz}, \mathrm{CDCl}_{3}\right) \delta: 2.26(\mathrm{~s}, 3 \mathrm{H}), 2.61-2.65(\mathrm{~m}, 2 \mathrm{H})$, 2.80-2.84 (m, 2H), $3.50(\mathrm{~s}, 2 \mathrm{H}), 3.79(\mathrm{~s}, 3 \mathrm{H}), 6.82-6.86(\mathrm{~m}, 2 \mathrm{H}), 7.16-$ $7.29(\mathrm{~m}, 7 \mathrm{H}) .{ }^{13} \mathrm{C}\left\{{ }^{1} \mathrm{H}\right\} \mathrm{NMR}\left(100 \mathrm{MHz}, \mathrm{CDCl}_{3}\right) \delta: 33.8,42.0,55.2,59.0$, $61.5,113.5,125.9,128.3,128.7,130.1,130.9,140.5,158.6$. IR $\left(v_{\max } / \mathrm{cm}^{-1}\right): 3061,3027,3000,2947$, 2835, 2788, 1677, 1610, 1509, 1454, 1242, 1172, 1035, 745, 698. HRMS (ESI+): calculated for [M+H] 256.1696, found: 256.1698 .

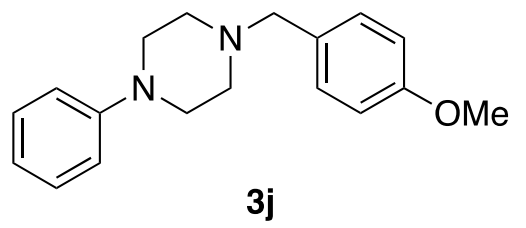

1-(4-Methoxybenzyl)-4-phenylpiperazine (3j): White solid. mp.: 108 ${ }^{\circ} \mathrm{C} .{ }^{1} \mathrm{H}$ NMR $\left(400 \mathrm{MHz}, \mathrm{CDCl}_{3}\right) \delta: 1.64(\mathrm{~s}, 1 \mathrm{H}), 2.57-2.61(\mathrm{~m}, 4 \mathrm{H})$, 3.17-3.21 (m, 4H), $3.51(\mathrm{~s}, 2 \mathrm{H}), 3.81(\mathrm{~s}, 3 \mathrm{H}), 6.83-6.93(\mathrm{~m}, 5 \mathrm{H})$, 7.23$7.28(\mathrm{~m}, 4 \mathrm{H}) .{ }^{13} \mathrm{C}\left\{{ }^{1} \mathrm{H}\right\} \mathrm{NMR}\left(100 \mathrm{MHz}, \mathrm{CDCl}_{3}\right) \delta: 49.1,53.0,55.3,62.5$, 113.6, 116.0, 119.6, 129.1, 130.0, 130.4, 151.4, 158.8. IR $\left(v_{\max } / \mathrm{cm}^{-1}\right): 3025,2986,2974,2955,2937$, 2920, 2877, 2816, 2765, 1611, 1596, 1507, 1451, 1442, 1299, 1239, 1140, 1030, 1008, 918, 831, 813, 762, 688. HRMS (ESI+): calculated for $[\mathrm{M}+\mathrm{H}]^{+}:$283.1805, found: 283.1805 .

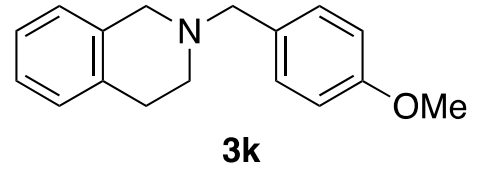

2-(4-Methoxybenzyl)-1,2,3,4-tetrahydroisoquinoline (3k): Yellow liquid. ${ }^{1} \mathrm{H}$ NMR $\left(400 \mathrm{MHz}, \mathrm{CDCl}_{3}\right) \delta: 2.72(\mathrm{t}, J=6.0 \mathrm{~Hz}, 2 \mathrm{H}), 2.86-2.90(\mathrm{~m}$, $2 \mathrm{H}), 3.61(\mathrm{~s}, 2 \mathrm{H}), 3.61(\mathrm{~s}, 2 \mathrm{H}), 3.80(\mathrm{~s}, 3 \mathrm{H}), 6.85-6.89(\mathrm{~m}, 2 \mathrm{H}), 6.96-6.98$ $(\mathrm{m}, 1 \mathrm{H}), 7.06-7.11(\mathrm{~m}, 3 \mathrm{H}), 7.28-7.32(\mathrm{~m}, 2 \mathrm{H}) .{ }^{13} \mathrm{C}\left\{{ }^{1} \mathrm{H}\right\} \mathrm{NMR}\left(100 \mathrm{MHz}, \mathrm{CDCl}_{3}\right) \delta:$ 29.1, 50.5, 55.2, $56.0,62.1,113.6,125.5,126.0,126.6,128.6,130.2,130.4,134.4,134.9,158.7$. IR $\left(v_{\max } / \mathrm{cm}^{-1}\right): 3063$, 
3022, 3003, 2912, 2833, 2795, 2754, 2721, 1674, 1611, 1510, 1244, 1035, 935, 744. HRMS (ESI+): calculated for $[\mathrm{M}+\mathrm{H}]^{+}:$254.1539, found: 254.1543 .

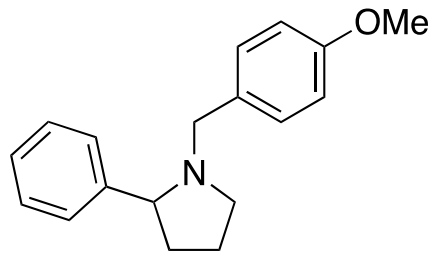

31

1-(4-Methoxybenzyl)-2-phenylpyrrolidine (31): White solid. mp.: $44{ }^{\circ} \mathrm{C}$. ${ }^{1} \mathrm{H}$ NMR (400 MHz, $\left.\mathrm{CDCl}_{3}\right) \delta: 1.67-1.92(\mathrm{~m}, 3 \mathrm{H}), 2.13-2.22(\mathrm{~m}, 2 \mathrm{H}), 2.98$ $(\mathrm{d}, J=12.6 \mathrm{~Hz}, 1 \mathrm{H}), 3.04-3.09(\mathrm{~m}, 1 \mathrm{H}), 3.33(\mathrm{t}, J=8.2 \mathrm{~Hz}, 1 \mathrm{H}), 3.77(\mathrm{~d}, J=$ $12.6 \mathrm{~Hz}, 1 \mathrm{H}), 3.78(\mathrm{~s}, 3 \mathrm{H}), 6.80-6.84(\mathrm{~m}, 2 \mathrm{H}), 7.16-7.20(\mathrm{~m}, 2 \mathrm{H}), 7.22-7.27$ $(\mathrm{m}, 1 \mathrm{H}), 7.32-7.37(\mathrm{~m}, 2 \mathrm{H}), 7.44-7.47(\mathrm{~m}, 2 \mathrm{H}) .{ }^{13} \mathrm{C}\left\{{ }^{1} \mathrm{H}\right\} \mathrm{NMR}(100 \mathrm{MHz}$, $\left.\mathrm{CDCl}_{3}\right) \delta: 22.3$ 35.2, 53.2, 55.2, 57.3, 69.4, 113.4, 126.9, 127.5, 128.4, 129.8, 131.8, 143.9, 158.4. IR $\left(v_{\max } / \mathrm{cm}^{-1}\right): 3068,3031,3000,2971,2950,2915,2875,2834,2790,2773,2718,2675,1609,1584,1508$, 1244, 1038, 811, 752, 696. HRMS (ESI+): calculated for $[\mathrm{M}+\mathrm{H}]^{+}: 268.1696$, found: 268.1693.

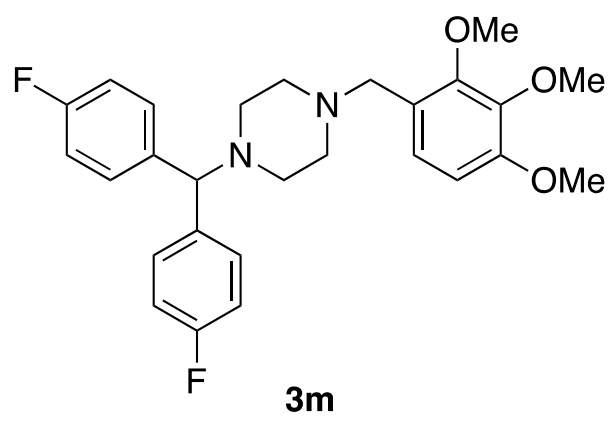

\section{1-(Bis(4-fluorophenyl)methyl)-4-(2,3,4,-trimethoxybenzyl)pi-} perazine $^{1}$ (3m; Lomerizine): Pale yellow viscous liquid. ${ }^{1} \mathrm{H}$ NMR (400 MHz, $\left.\mathrm{CDCl}_{3}\right) \delta:$ 2.36-2.48 (m, 8H), 3.47 (s, 2H), 3.84 (s, 3H), $3.86(\mathrm{~s}, 3 \mathrm{H}), 3.87(\mathrm{~s}, 3 \mathrm{H}), 4.19(\mathrm{~s}, 1 \mathrm{H}), 6.61(\mathrm{~d}, J=8.7 \mathrm{~Hz}$, 1H), 6.92-6.98 (m, 5H), 7.29-7.35 (m, 4H). ${ }^{13} \mathrm{C}\left\{{ }^{1} \mathrm{H}\right\}$ NMR (100

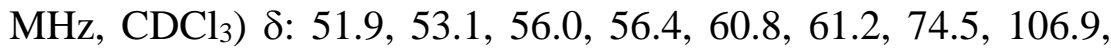

$115.3\left(\mathrm{~d},{ }^{2} J_{\mathrm{C}-\mathrm{F}}=21.3 \mathrm{~Hz}\right), 124.0,125.2,129.2\left(\mathrm{~d},{ }^{3} J_{\mathrm{C}-\mathrm{F}}=7.4 \mathrm{~Hz}\right), 138.4\left(\mathrm{~d},{ }^{4} J_{\mathrm{C}-\mathrm{F}}=3.3 \mathrm{~Hz}\right), 142.2,152.6$, 152.8, $161.7\left(\mathrm{~d},{ }^{1} J_{\mathrm{C}-\mathrm{F}}=245.0 \mathrm{~Hz}\right) . \mathrm{IR}\left(v_{\max } / \mathrm{cm}^{-1}\right): 3065,3040,2995,2937,2879,2809,2766,1602,1504$, 1495, 1464, 1416, 1278, 1220, 1094, 1005, 825. HRMS (ESI+): calculated for $[\mathrm{M}+\mathrm{H}]^{+}: 469.2297$, found: 469.2298 . 
<smiles>Cc1cc(C)cc([C@H]2CCCN2Cc2ccc(Oc3ccc(C(N)=O)cc3F)cc2)c1</smiles>

$(S)-3 n$

4-(4-(((S)-2-(3,5-Dimethylphenyl)pyrrolidin-1-yl)methyl)phenoxy)-3-fluorobenzamide ${ }^{2} \quad((S)-3 n$; Aticaprant):

Pale yellow viscous liquid. ${ }^{1} \mathrm{H}$ NMR $\left(400 \mathrm{MHz},\left(\mathrm{D}_{3} \mathrm{C}\right)_{2} \mathrm{SO}\right) \delta$ : 1.52-1.62 (m, 1H), 1.70-1.81 (m, 2H), 2.07-2.18 (m, 2H), 2.26 $(\mathrm{s}, 6 \mathrm{H}), 2.50-2.52(\mathrm{~m}, 1 \mathrm{H}), 2.94-2.99(\mathrm{~m}, 1 \mathrm{H}), 3.03(\mathrm{~d}, J=13.0$ $\mathrm{Hz}, 1 \mathrm{H}), 3.28$ (t, $J=8.2 \mathrm{~Hz}, 1 \mathrm{H}), 3.66(\mathrm{~d}, J=13.0 \mathrm{~Hz}, 1 \mathrm{H})$, $6.87(\mathrm{~s}, 1 \mathrm{H}), 6.99$ (d, $J=8.2 \mathrm{~Hz}, 2 \mathrm{H}), 7.03(\mathrm{~s}, 2 \mathrm{H}), 7.09(\mathrm{t}, J=8.5 \mathrm{~Hz}, 1 \mathrm{H}), 7.27(\mathrm{~d}, J=8.7 \mathrm{~Hz}, 2 \mathrm{H}), 7.49$ $(\mathrm{s}, 1 \mathrm{H}), 7.72-7.74(\mathrm{~m}, 1 \mathrm{H}), 7.87(\mathrm{dd}, J=12.1,1.9 \mathrm{~Hz}, 1 \mathrm{H}), 8.04(\mathrm{~s}, 1 \mathrm{H}) .{ }^{13} \mathrm{C}\left\{{ }^{1} \mathrm{H}\right\} \mathrm{NMR}(100 \mathrm{MHz}$, $\left.\left(\mathrm{D}_{3} \mathrm{C}\right)_{2} \mathrm{SO}\right) \delta: 21.0,21.9,34.8,52.8,56.7,68.7,116.3\left(\mathrm{~d},{ }^{2} J_{\mathrm{C}-\mathrm{F}}=18.8 \mathrm{~Hz}\right), 117.7,120.2,124.8,125.0$, $128.4,130.0,130.7\left(\mathrm{~d},{ }^{3} J_{\mathrm{C}-\mathrm{F}}=5.7 \mathrm{~Hz}\right), 135.3,137.3,143.5,146.1\left(\mathrm{~d},{ }^{2} J_{\mathrm{C}-\mathrm{F}}=11.5 \mathrm{~Hz}\right), 152.5\left(\mathrm{~d},{ }^{1} J_{\mathrm{C}-\mathrm{F}}=\right.$ 246.6 Hz), 154.6, 166.0. IR $\left(v_{\max } / \mathrm{cm}^{-1}\right): 3353,3211,2964,2918,2871,2787,1660,1604,1581,1502$, $1435,1385,1274,1213,1161,1095,848$. HRMS (ESI+): calculated for $[\mathrm{M}+\mathrm{H}]^{+}:$419.2129, found: 419.2128. $[\alpha]_{\mathrm{D}}{ }^{20}-21.1(c=0.50, \mathrm{EtOH})$. Chiral HPLC (ChiralPak AD-H, $\phi 4.6 \mathrm{~mm} \times \mathrm{L} 250 \mathrm{~mm}$, heptane:EtOH:MeOH:N,N-dimethylethylamine = 80:10:10:0.2, $0.6 \mathrm{~mL} / \mathrm{min}, \lambda=270 \mathrm{~nm}): t_{\mathrm{R}} / \mathrm{min}=7.6(99 \%)$, $13.0(1 \%)$.

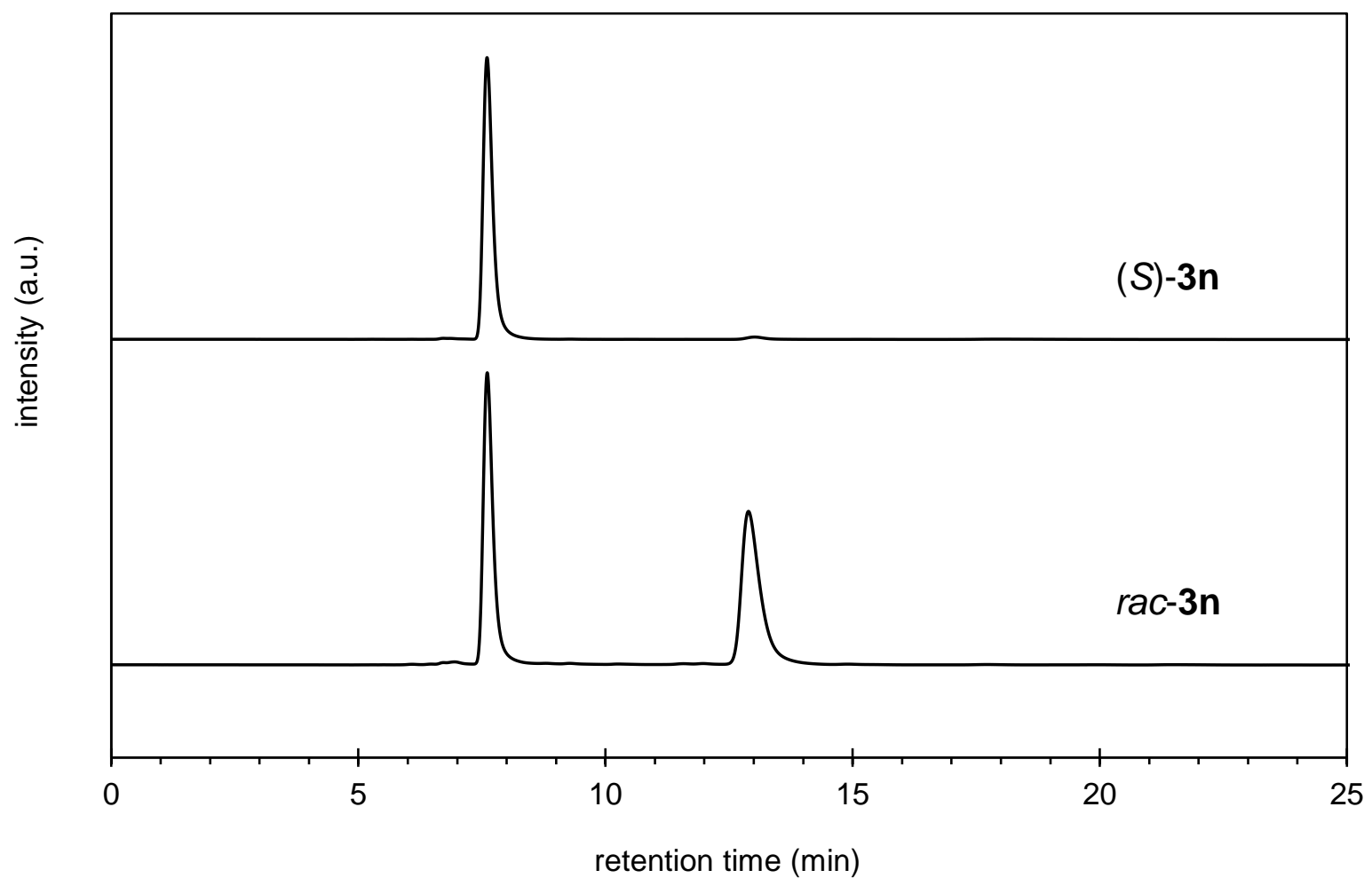

Figure S3. Chiral HPLC charts of Aticaprant ((S)-3n) and rac-3n. 


\section{References}

(1) Murai, T.; Ui, K.; Narengerile. Sequential Addition Reactions of Two Molecules of Grignard Reagents to Thioformamides. J. Org. Chem. 2009, 74, 5703.

(2) Mitch, C. H.; Quimby, S. J.; Diaz, N.; Pedregal, C.; de la Torre, M. G.; Jimenez, A.; Shi, Q.; Canada, E. J.; Kahl, S. D.; Statnick, M. A.; McKinzie, D. L.; Benesh, D. R.; Rash, K. S.; Barth, V. N. Discovery of aminobenzyloxyarylamides as $\kappa$ opioid receptor selective antagonists: application to preclinical development of a $\kappa$ opioid receptor antagonist receptor occupancy tracer. J. Med. Chem. 2011, 54, 8000. 